\title{
Calderón Stabilized Time Domain Integral Equation Solvers
}

\author{
Francesco P. Andriulli*1, Kristof Cools ${ }^{2}$, Femke Olyslager ${ }^{2}$, and Eric \\ Michielssen $^{1}$ \\ ${ }^{1}$ Department of Electrical Engineering and Computer Science \\ University of Michigan at Ann Arbor \\ ${ }^{2}$ Department of Information Technology (INTEC), Ghent University
}

\section{Introduction}

Marching on in time (MOT)-based time domain integral equation (TDIE) solvers represent an increasingly appealing avenue for analyzing transient electromagnetic interactions with large and complex structures. Compared to their differential equation counterparts, MOT-TDIE solvers automatically impose radiation conditions, do not require unknown fields to be discretized throughout homogeneous volumes, and are highly immune to numerical dispersion. In addition, the use of averaging, exact integration, Helmholtz decomposition, and combined field integral equation methods have rendered these solvers stable for all practical purposes (see [1] and references therein). Here, we present new techniques for stabilizing MOT-TDIE solvers that distinguish themselves from the above ones in that they operate hand in glove with recently developed Calderon-based MOT-TDIE preconditioners. The proposed preconditioners provide simple mechanisms for eliminating "resonant" and "DC" eigenvalues from combined and electric field TDIEs, thereby rendering them immune from late-time ringing and non-oscillatory growth of their solutions. $\mathrm{Nu}-$ merical results that show the validity and the effectiveness of the proposed equations are presented.

\section{Formulation}

Let $\Gamma$ and $\hat{\boldsymbol{n}}_{\boldsymbol{r}}$ denote the surface of a perfect electrically conducting smooth object and its outward pointing unit normal at $\boldsymbol{r}$, respectively. Assume that $\Gamma$ resides in a homogeneous medium with electric permittivity $\epsilon$ and magnetic permeability $\mu$, and is illuminated by the electromagnetic field $\left(\boldsymbol{E}^{i}(\boldsymbol{r}, t), \boldsymbol{H}^{i}(\boldsymbol{r}, t)\right)$. The current density $\boldsymbol{J}(\boldsymbol{r}, t)$ induced on $\Gamma$ in response to this excitation satisfies the time domain EFIE $[1]$

$$
\frac{\partial \mathcal{T}(\boldsymbol{J})}{\partial t}=\frac{\partial\left(\mathcal{T}_{s}+\mathcal{T}_{h}\right)(\boldsymbol{J})}{\partial t}=-\hat{\boldsymbol{n}}_{\boldsymbol{r}} \times \frac{\partial \boldsymbol{E}^{i}(\boldsymbol{r}, t)}{\partial t}
$$

where

$$
\begin{aligned}
& \mathcal{T}_{h}(\boldsymbol{J})=\left(\hat{\boldsymbol{n}}_{\boldsymbol{r}} \times \nabla \mathcal{R}\left(\int_{0}^{t} \frac{\nabla_{s} \cdot \boldsymbol{J}}{\epsilon} \mathrm{d} t\right)\right) \\
& \mathcal{T}_{s}(\boldsymbol{J})=-\left(\hat{\boldsymbol{n}}_{\boldsymbol{r}} \times \mathcal{R}\left(\mu \frac{\partial \boldsymbol{J}}{\partial t}\right)\right) .
\end{aligned}
$$

with $\mathcal{R}(f)=\int_{\Gamma} \frac{f\left(\boldsymbol{r}^{\prime}, t-\left|\boldsymbol{r}-\boldsymbol{r}^{\prime}\right| / c\right)}{4 \pi\left|\boldsymbol{r}-\boldsymbol{r}^{\prime}\right|} \mathrm{d} \boldsymbol{r}^{\prime}$. The temporal differentiations in (1) undo the inconvenient temporal integration in (2). To numerically solve (1), $\Gamma$ is approximated by a mesh of planar triangles, and the current density $\boldsymbol{J}(\boldsymbol{r}, t)$ is approximated as

$$
\boldsymbol{J}(\boldsymbol{r}, t) \approx \sum_{j=1}^{N_{t}} \sum_{n=1}^{N_{s}} J_{j, n} \boldsymbol{f}_{n}(\boldsymbol{r}) T_{j}(t)
$$


where $\boldsymbol{f}_{n}(\boldsymbol{r}), n=1, \ldots, N_{s}$ are Rao-Wilton-Glisson basis functions defined on the mesh's $N_{s}$ interior edges and $T_{j}(t), j=1, \ldots, N_{t}$ are higher-order polynomial interpolants [1] satisfying $T_{j}(t)=T(t-j \Delta t)$ with $T(t)=0 \forall t<\Delta t ; \Delta t$ denotes the time step size. Using expansion (4) in (1) and spatial Galerkin testing the resulting equation at time $t_{j}=j \Delta t$ yields

$$
\mathrm{TJ}=\mathrm{E}
$$

where

$$
\begin{aligned}
& \mathbf{T}=\left(\begin{array}{cccc}
\mathbf{T}_{0} & & & \\
\mathbf{T}_{1} & \mathbf{T}_{0} & & \\
\mathbf{T}_{2} & \mathbf{T}_{1} & \mathbf{T}_{0} & \\
\vdots & \vdots & \vdots & \ddots
\end{array}\right), \mathrm{J}=\left(\begin{array}{c}
\mathrm{J}_{0} \\
\mathrm{~J}_{1} \\
\mathrm{~J}_{2} \\
\vdots
\end{array}\right), \mathrm{E}=\left(\begin{array}{c}
\mathrm{E}_{0} \\
\mathrm{E}_{1} \\
\mathrm{E}_{2} \\
\vdots
\end{array}\right),\left\{\mathrm{J}_{j}\right\}_{n}=J_{j, n} \\
& \left\{\mathbf{T}_{k}\right\}_{m, n}=\left.\left\langle\boldsymbol{f}_{m}(\boldsymbol{r}), \frac{\partial \mathcal{T}\left(T_{k} \boldsymbol{f}_{n}\right)}{\partial t}\right\rangle\right|_{t=0}, \quad\left\{\mathrm{E}_{j}\right\}_{n}=\left.\left\langle\boldsymbol{f}_{n}(\boldsymbol{r}),-\hat{\boldsymbol{n}}_{\boldsymbol{r}} \times \frac{\partial \boldsymbol{E}^{i}(\boldsymbol{r}, t)}{\partial t}\right\rangle\right|_{t=t_{j}}
\end{aligned}
$$

with $\langle a(\boldsymbol{r}, t), b(\boldsymbol{r}, t)\rangle=\int_{\Gamma} a(\boldsymbol{r}, t) b(\boldsymbol{r}, t) \mathrm{d} \boldsymbol{r}$. A similar discretization can be performed for the time domain Magnetic Field Integral Equation (MFIE) $\boldsymbol{J} / 2+\mathcal{K}(\boldsymbol{J}(\boldsymbol{r}, t))=$ $\hat{\boldsymbol{n}}_{\boldsymbol{r}} \times \boldsymbol{H}^{i}(\boldsymbol{r}, t)$ where $\mathcal{K}(\boldsymbol{J})=\frac{1}{2}(\hat{\boldsymbol{n}} \times \nabla \times \mathcal{R}(\boldsymbol{J}))$ yielding

$$
\mathrm{KJ}=\mathrm{H}
$$

Equation (5) (and similarly (6)) can be cast in Marching-On-in-Time (MOT) form as

$$
\mathbf{T}_{0} \mathrm{~J}_{j}=\mathrm{E}_{j}-\sum_{k=0}^{j-1} \mathbf{T}_{k} \mathrm{~J}_{j-k}, \quad j \geq 0 .
$$

This equation can be solved for the current coefficient vector $J_{j}$ given the current coefficient vectors $\mathrm{J}_{k}, k=0, \ldots, j-1$. The MOT process may be unstable, i.e. produces erroneous solutions $\mathrm{J}_{k}$ that exponentially grow for increasing $k$. The stability of the solution is governed by the polynomial spectrum [3] of (5) which is defined as the (ordinary) spectrum of the matrix

$$
\mathbf{T}^{p}=\left(\begin{array}{cccc}
\left(\mathbf{T}_{0}\right)^{-1} \mathbf{T}_{1} & \left(\mathbf{T}_{0}\right)^{-1} \mathbf{T}_{2} & \left(\mathbf{T}_{0}\right)^{-1} \mathbf{T}_{3} & \ldots \\
\mathbf{I} & \mathbf{0} & \cdots & \\
\mathbf{0} & \mathbf{I} & \mathbf{0} & \ldots \\
\vdots & \ddots & \ddots &
\end{array}\right)
$$

The MOT solution of (5) is stable when the spectrum of $\mathbf{T}^{p}$ resides strictly inside the unit circle of the complex plane [3]. The numerical error introduced by the discretization may shift some polynomial eigenvalues of (5) that should reside on the unit circle out of the unit circle. These polynomial eigenvalues belong to two classes: "resonant" eigenvalues of the form $\lambda=e^{i \theta}$ with $\theta \neq 0$ and "DC" (degenerate) eigenvalues $\lambda=1$.

Resonant eigenvalues. The eigenvalues $\lambda=e^{i \theta}(\theta \neq 0)$ in the polynomial spectrum of (5) are due to the spurious interior resonances characterizing the solutions of (1). These resonances can be eliminated using the time domain Combined Field Integral Equation (CFIE) [4]. Unfortunately the CFIE is ill-conditioned in the presence of dense discretizations. The use of Calderón techniques allows us to obtain a time domain integral equation free from resonances and that is well-conditioned independent of the discretization. The use of time domain Calderón formulas [2] led 


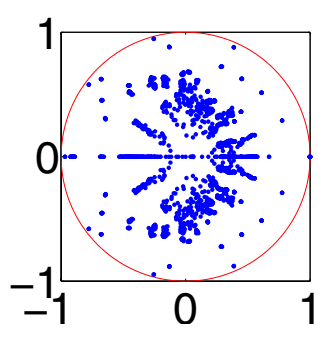

(a) EFIE

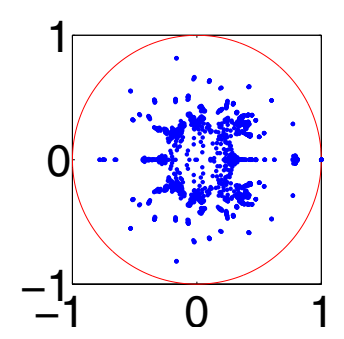

(b) pcCFIE

Figure 1: Polynomial eigenvalues of EFIE and of pcCFIE. In the figures the circle is the unit ball in the complex plane centered in $0+0 \mathrm{i}$.

us to adopt the matrix $\mathbf{T G}^{-1}$ as a preconditioner for the matrix $\mathbf{T}$, where $\mathbf{G}$ is a properly chosen Gram matrix. A similar approach leads to the following preconditioned combined field integral equation (pcCFIE)

$$
\left(\mathbf{T}_{l o c} \mathbf{G}^{-1} \mathbf{T}+\mathbf{K}\right) \mathbf{J}=\mathbf{T}_{l o c} \mathbf{G}^{-1} \mathrm{E}+\mathrm{H}
$$

with

$$
\mathbf{T}_{l o c}=\left(\begin{array}{cccc}
\mathbf{T}_{0} & & & \\
& \mathbf{T}_{0} & & \\
& & \mathbf{T}_{0} & \\
& & & \ddots
\end{array}\right) .
$$

The presence of a localized EFIE matrix $\mathbf{T}_{l o c}$ makes equation (9) a valid combined field integral equation. The need for localizing the square of the EFIE operator is a well known and delicate issue in designing well-conditioned and accurate frequency domain EFIEs. It is remarkable that in the time domain this problem has a natural solution. The validity of the localization in $\mathbf{T}_{l o c}$ is due to the fact that $\mathbf{T}_{l o c} \mathbf{G}^{-1} \mathbf{T}$ shares with $\mathbf{T G}^{-1} \mathbf{T}$ its ordinary spectrum and with $\mathbf{T}$ its polynomial spectrum. The new pcCFIE has been tested on a sphere of radius $0.25 \mathrm{~m}$ discretized using 255 unknowns. The incident wave is a Gaussian $\boldsymbol{E}^{i}(\boldsymbol{r}, t)=4 \hat{\boldsymbol{x}} e^{-\gamma^{2}} /(T \sqrt{\pi})$ with $\gamma=4\left(c t-c t_{0}-\hat{\boldsymbol{z}} \cdot \boldsymbol{r}\right) / T, T=200$ meter, and $t_{0}=300$ lightmeter. Fig. 1(a) shows the polynomial spectrum of the EFIE plotted on the complex plane, Fig. 1(b) shows the polynomial spectrum of the pcCFIE: the resonant eigenvalues of the EFIE disappeared from the unitary circle of the pcCFIE. Fig. 3(a) compares the surface currents obtained using both equations.

DC eigenvalues. The presence of eigenvalues $\lambda=1$ in the polynomial spectrum of (5) stems from the fact that static current loops reside in the kernel of $\mathcal{T}$. A proper discretization of the square EFIE operator $\mathcal{T}^{2}$ overcomes the problem. In fact, defining

$$
\begin{aligned}
& \tilde{\mathcal{T}}_{h}(\boldsymbol{J})=\left(\hat{\boldsymbol{n}} \times \nabla \mathcal{R}\left(\frac{\nabla_{s} \cdot \boldsymbol{J}}{\epsilon}\right)\right) \\
& \tilde{\mathcal{T}}_{s}(\boldsymbol{J})=-(\hat{\boldsymbol{n}} \times \mathcal{R}(\mu \boldsymbol{J})) .
\end{aligned}
$$

it is easy to see that $\tilde{\mathcal{T}}_{h} \tilde{\mathcal{T}}_{s}=\mathcal{T}_{h} \mathcal{T}_{s}$ and $\tilde{\mathcal{T}}_{s} \tilde{\mathcal{T}}_{h}=\mathcal{T}_{s} \mathcal{T}_{h}$. It follows that the operator $\mathcal{T}^{2}$ can be expressed as $\mathcal{T}^{2}=\mathcal{T}_{s}^{2}+\mathcal{T}_{s} \mathcal{T}_{h}+\mathcal{T}_{h} \mathcal{T}_{s}+\mathcal{T}_{h}^{2}=\mathcal{T}_{s}^{2}+\tilde{\mathcal{T}}_{s} \tilde{\mathcal{T}}_{h}+\tilde{\mathcal{T}}_{h} \tilde{\mathcal{T}}_{s}$. By leveraging the properties of the Laplacian, it can be shown that $\tilde{\mathcal{T}}_{h} \tilde{\mathcal{T}}_{s}$ resolves current loops of any temporal signature. Moreover the definitions in (10)-(11) do not contain time integrals and, consequently, can be discretized without additional time 


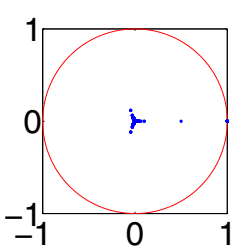

(a) EFIE

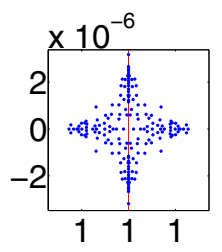

(b) EFIE, zoom in near

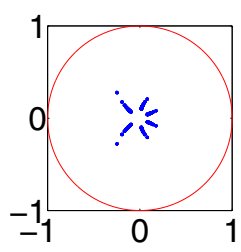

(c) pcEFIE

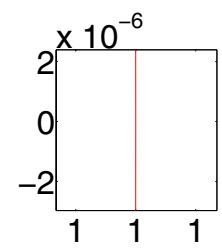

(d) pcEFIE, zoom in near $1+0 \mathrm{i}$

Figure 2: Polynomial eigenvalues of EFIE and of pcEFIE. Comparing (b) and (d) note the absence of eigenvalues in the latter.

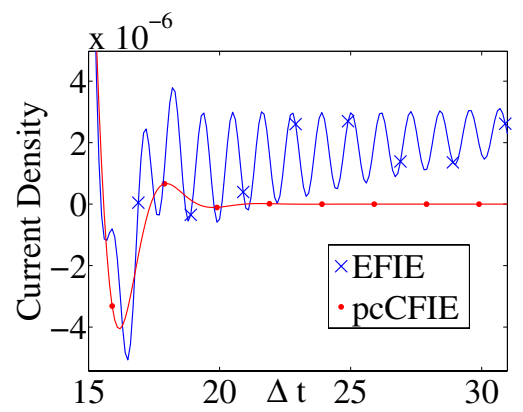

(a) comparison pcCFIE-EFIE

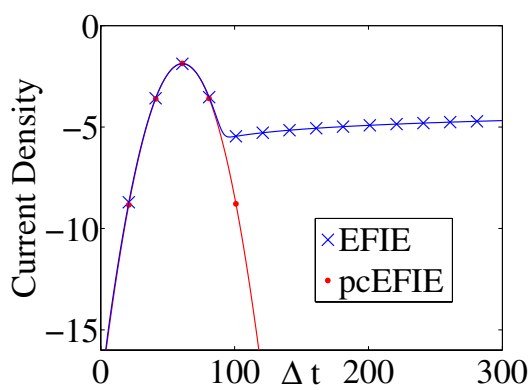

(b) comparison pcEFIE-EFIE

Figure 3: Surface currents $([\mathrm{A} / \mathrm{m}])$ on a sphere as a function of the time step $\Delta t$ ([lightmeter]) obtained using different equations.

differentiation. Therefore the preconditioned EFIE (pcEFIE) proposed is

$$
\tilde{\mathbf{T}}_{s} \mathbf{G}^{-1} \tilde{\mathbf{T}}_{h}+\tilde{\mathbf{T}}_{h} \mathbf{G}^{-1} \tilde{\mathbf{T}}_{s}+\mathbf{T}_{s} \mathbf{G}^{-1} \mathbf{T}_{s}=\mathbf{T E}
$$

The pcEFIE has been tested on the same example used to validate the pcCFIE. The polynomial spectra of the EFIE and pcEFIE are plotted in Fig. 2. Note that the eigenvalues around 1 of the EFIE (Fig. 2(b)) are not in the polynomial spectrum of the pcEFIE (Fig. 2(d)). The surface currents on the sphere obtained with the EFIE and with the pcEFIE are depicted in Fig. 3(b): clearly the pcEFIE solution cannot suffer from DC instabilities.

\section{References}

[1] A. A. Ergin, B. Shanker, and E. Michielssen, "Analysis of transient wave scattering from rigid bodies using a Burton-Muller approach," J. Acoust. Soc. Am., vol. 106, pp. 2396-2404, 1999.

[2] K. Cools, F. P. Andriulli, and E. Michielssen, "Time-Domain Calderón Identities and Preconditioning of the Time-Domain EFIE," IEEE Antennas and Propagation Society International Symposium, pp. 2975-2978, July 2006.

[3] S. P. Walker, M. J. Bluck, and I. Chatzis, "The stability of integral equation time domain computations for three-dimensional scattering "Int. J. Numer. Model., vol. 15, pp. 459-474, 2000.

[4] B. Shanker, A. Ergin, K. Aygun, and E. Michielssen, "Analysis of transient electromagnetic scattering from closed surfaces using a combined field integral equation," IEEE Trans. Antennas Propagation, vol. 48, no. 7, pp. 1064-1074, 2000 . 


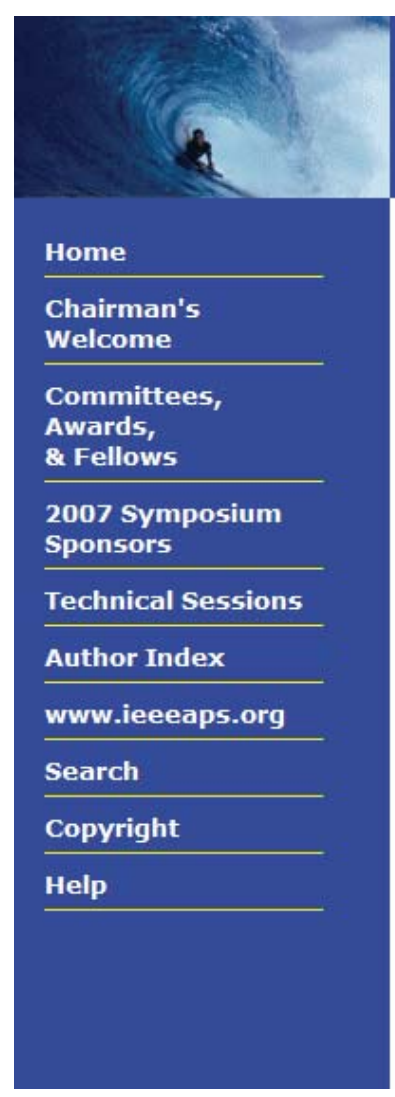

2007 IEEE Antennas and Propagation Society International Symposium

Sheraton Waikiki | Honolulu, Hawaii, USA | June 10-15, 2007
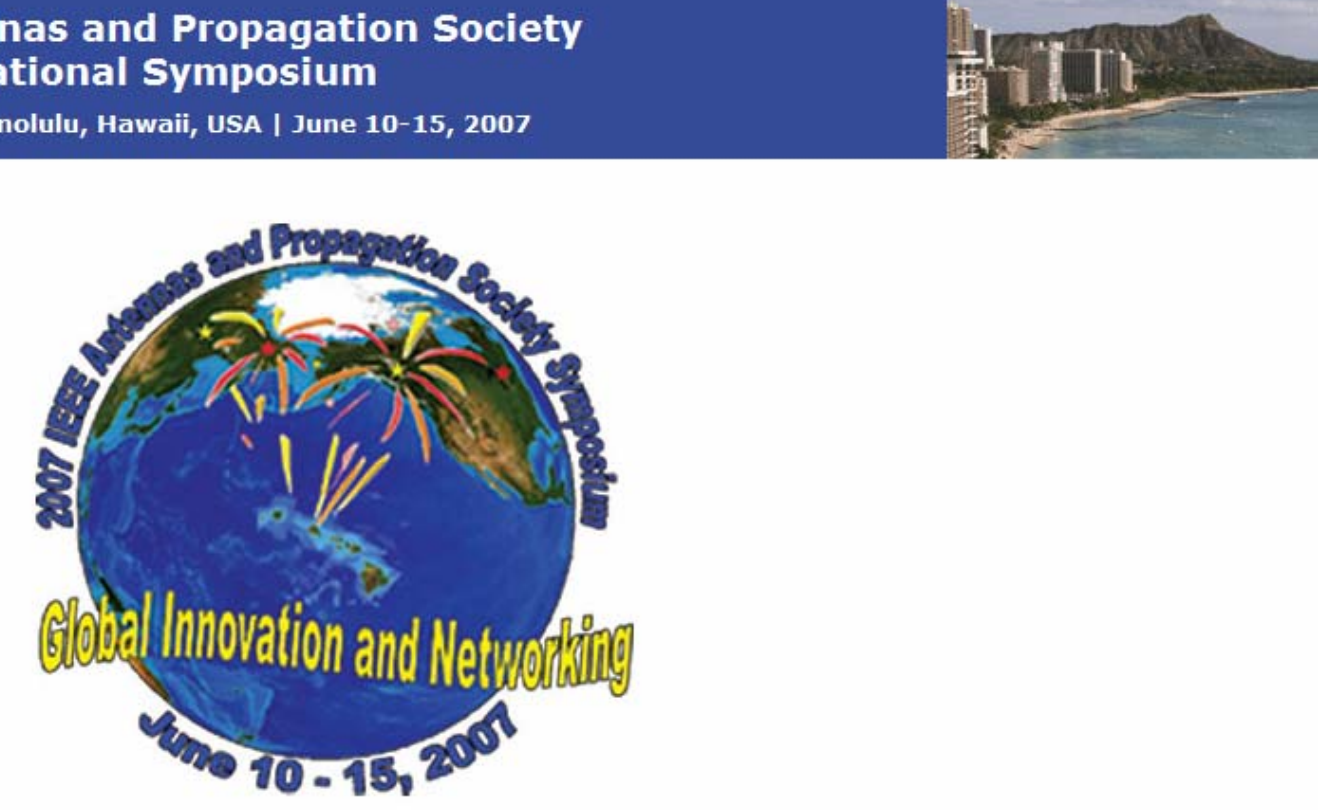

\section{- IEEE}

$(A / 5)$ 